\title{
Spin switching in antiferromagnets using Néel-order spin-orbit
}

\author{
torques* \\ P. Wadley and K. W. Edmonds ${ }^{\dagger}$ \\ School of Physics and Astronomy, University of Nottingham, University Park, \\ Nottingham NG7 2RD, United Kingdom
}

\begin{abstract}
Antiferromagnets offer considerable potential for electronic device applications. This article reviews recent demonstrations of spin manipulation in antiferromagnetic devices using applied electrical currents. Due to spin-orbit coupling in environments with particular crystalline or structural symmetries, the electric current can induce an effective magnetic field with a sign that alternates on the lengthscale of the unit cell. The staggered effective field provides an efficient mechanism for switching antiferromagnetic domains and moving antiferromagnetic domain walls, with writing speeds in the terahertz regime.
\end{abstract}

spintronics, antiferromagnetism, current-induced torques, magnetic domains

PACS:72.15.Gd, 75.50.Ee, 75.60.Ch, 85.75.-d

\section{Introduction}

The development of spintronic devices based on ferromagnetic (FM) metals has had a huge impact on information and communication technologies. Since the introduction of thin film magnetoresistive read heads in the mid-1990s, hard disk storage capacities have increased by several orders of magnitude. Ferromagnetic tunnel junctions form the bits of magnetic random access memories (MRAM), which offer advantages of nonvolatility, stability and high speed compared to competing semiconductor-based technologies. Antiferromagnetic (AF) materials, in which the local magnetic moment vectors of neighbouring atoms sum to zero, play a key, albeit passive, role in such devices: exchange coupling at the interface between FM and AF layers causes the FM layer to become stable against external magnetic fields, providing a fixed reference layer in a spin-valve structure. This important application has stimulated extensive research over the last two decades on exchange bias and other interface-induced phenomena at FM-AF interfaces ${ }^{[1]}$.

AF materials have not yet found application as active components in spintronic devices, for two principal reasons. Firstly, the cancellation of neighbouring magnetic moments results in zero stray magnetic field, making their magnetic properties hard to measure. Secondly, AF materials are insensitive to external fields of typically several tesla, making them hard to control. However, neither the stray magnetic field nor the

\footnotetext{
${ }^{*}$ Project supported by EPSRC (grant EP/P019749/1). P.W. acknowledges support from the Royal Society through a University Research Fellowship.

${ }^{+}$Corresponding author. E-mail: Kevin.Edmonds@ @ottingham.ac.uk
} 
sensitivity of external magnetic fields of FM layers are used in modern MRAM devices: information is read electrically using tunnel magnetoresistance, and electrical currentinduced spin torques provide the writing mechanism. Therefore, these historic advantages of FM materials actually become a hindrance in MRAM, as the stray fields must be compensated to prevent unwanted cross-talk between neighbouring magnetic elements. It has been demonstrated that large tunnelling anisotropic magnetoresistance can be observed in tunnel junctions with an AF layer as the active element ${ }^{[2]}$, providing an important proof of the principle that AF materials can provide the required electrical readout signals in a memory device. The recent demonstrations of electrical control of AF order ${ }^{[3-12]}$, described in detail in the present review article, firmly establish the prospects for fully readable and writable AF devices.

Antiferromagnets offer a further important advantage over ferromagnets for spintronic device applications, in terms of the speed of operation. The limiting timescale for writing the magnetization state is set by the magnetic resonance frequency which is of the order of $\mathrm{GHz}$ in FM materials. On the other hand, the resonance frequency in AF materials is enhanced by the strong antiparallel coupling of neighbouring moments, and is typically in the $\mathrm{THz}$ range ${ }^{[13]}$. Hence, rapid reorientation of AF moments on a timescale of a few picoseconds is possible ${ }^{[14]}$.

This article presents a focussed review of recent demonstrations of electrical switching in AF thin films using current-induced spin-orbit torques. For wider reviews of the rapidly developing field of AF spintronics, including spin transport, dynamical properties, opto-spintronics and topological effects, the reader is referred to the recent focus issue of Nature Physics ${ }^{[15]}$ and other recent extensive reviews ${ }^{[16,17]}$. In the present review, Section 2 gives a brief overview of current-induced torques that arise in due to spin-orbit coupling in FM and AF materials. Section 3 describes the structure and properties of CuMnAs, a material which offers favourable characteristics for utilization in AF memory devices. This has been the focus of several recent studies of currentinduced switching of AF order, including the first direct experimental observations ${ }^{[3]}$, as well as high speed (up to THz) switching ${ }^{[6]}$, as described in section 4 . Section 5 collects together related studies in other material systems, particularly $\mathrm{Mn}_{2} \mathrm{Au}$ and $\mathrm{Pt} / \mathrm{NiO}$. A summary and outlook is given in section 6 .

\section{Spin-orbit torques in ferromagnets and antiferromagnets}

In systems with broken inversion symmetry, due to spin-orbit coupling the carrier momentum is correlated with the spin angular momentum; carriers propagating in opposite directions through the crystal lattice will have opposite direction of spin polarization. Hence, an electric current can induce a non-equilibrium spin polarization which, in a magnetic material, can induce a torque on the local magnetic moments. As illustrated in Fig. 1(a) and Fig. 1(b), this spin-orbit torque (SOT) can have two distinct components: a component with the form of a current-induced effective magnetic field (field-like torque),

$$
T_{F L} \sim \boldsymbol{m} \times \sigma,
$$


where $\boldsymbol{m}$ is the local magnetic moment, and $\sigma$ is the current-induced spin polarization; and a component with the same form as the phenomenological damping term of the Landau-Lifschitz-Gilbert equation describing magnetization dynamics (damping-like torque):

$$
T_{D L} \sim \boldsymbol{m} \times(\boldsymbol{m} \times \sigma) .
$$

The inversion asymmetry giving rise to the non-equilibrium spin polarization can be either due to the bulk crystal structure (Dresselhaus effect) ${ }^{[18,19]}$, or can be due to the built-in internal electric field at the surfaces or interfaces of materials, such as in heavy metal / FM bilayers (Rashba effect) ${ }^{[20,21]}$. In such structures, including Pt/Co and $\mathrm{Ta} / \mathrm{CoFeB}$, the part of the current flowing through the heavy metal layer induces a transverse spin current due to the spin Hall effect, and its absorption by the FM produces a damping-like torque ${ }^{[22]}$. It has been shown that the damping-like SOT due to either the Rashba effect or the spin Hall effect can induce magnetization switching in 3terminal MRAM elements ${ }^{[20,23,24]}$. However, to ensure deterministic switching into a fixed direction determined by the current, either an external magnetic field or some other mechanism to break the in-plane symmetry is required in addition to the dampinglike SOT $\underline{[25-28]}$.

In an antiferromagnet, a current-induced spin polarization due to inversion asymmetry can in principle act on the local magnetic moments through the same mechanisms as in ferromagnets. In the case of a field-like SOT in an AF material (Fig. 1(c)), the effective magnetic field competes against the inter-sublattice exchange coupling, with typical magnitude of several hundreds of teslas. Consequently, only a small canting of the AFcoupled moments can occur for realistic current densities. In contrast, the damping-like SOT acts with the same handedness on each sublattice (Fig. 1(d)), so can trigger a precession of the local magnetic moments while maintaining their antiferromagnetic configuration ${ }^{[29]}$.

The extra degree of freedom offered by the magnetic sublattice symmetry in AFs enables a type of SOT which does not have a direct counterpart in FMs. For a centrosymmetric crystal, the net spin polarization generated by an electrical current is zero. However, the local spin polarization is determined by the symmetry of the individual atomic sites, rather than the global crystal symmetry ${ }^{[30]}$. If the magnetic sublattices of an AF are inversion partners of a centrosymmetric crystal, an electric current can induce a local spin polarization which alternates in sign between the two sublattices (Fig. 1(e)). The current-induced local spin polarization can couple strongly to the local magnetic moments to produce a Néel-order spin-orbit torque (NSOT), enabling a current-induced rotation of the local moments while preserving their AF order ${ }^{[31,32]}$. The NSOT can produce a fast and controllable switch of the AF order between two orthogonal crystal axes, which could be induced by, e.g., current pulses along two orthogonal axes (Fig. 1(f)). A full $180^{\circ}$ reversal of the sublattice magnetization is in principal also possible, for a system with a uniaxial easy axis at angle $\delta$ to the current direction, where $0<\delta<\pi / 2$. In the latter case, the reversal can be 
achieved by reversing the current polarity ${ }^{[33]}$.

Current-induced motion of antiferromagnetic domain walls has been investigated both for damping-like torques ${ }^{[34]}$ and Néel-order field-like torques ${ }^{[35]}$. A common feature of both studies is that the speed of the domain wall motion can be much larger in AFs than in FMs. In a FM material, the domain wall speed is limited by the so-called Walker breakdown, where the wall motion changes from a steady flow to an oscillatory behaviour associated with the precession of the wall magnetization ${ }^{[36]}$. In an AF material, the precession is opposed by the strong inter-sublattice exchange coupling, and the wall speed is limited only by the (much larger) spin wave velocity. Hence, speeds of several $10 \mathrm{~s}$ of $\mathrm{km} / \mathrm{s}$ are predicted, compared to limiting speeds of typically $10-100 \mathrm{~m} / \mathrm{s}$ for FM walls ${ }^{[35]}$. The antiferromagnetic exchange coupling also results in a very small domain wall inertia, favouring current-induced domain wall motion for ultra-short ( $\sim$ ps) current pulses ${ }^{[35]}$.
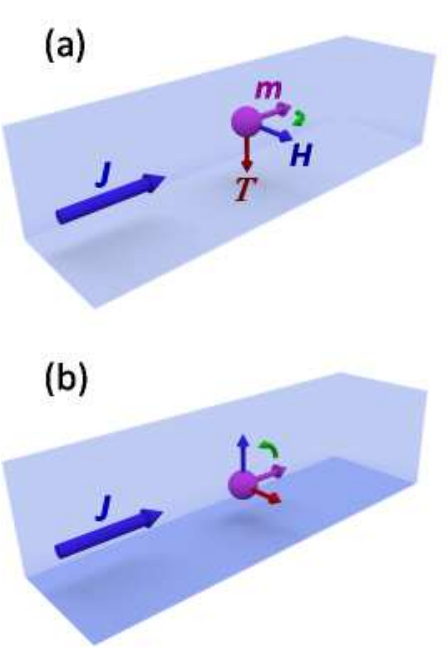

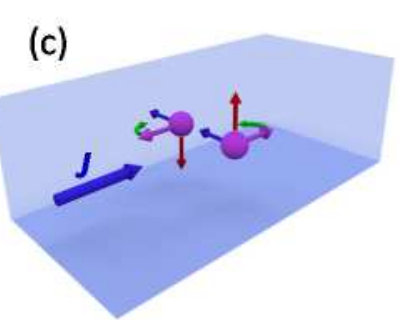

(d)

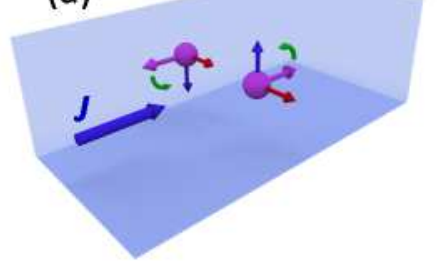

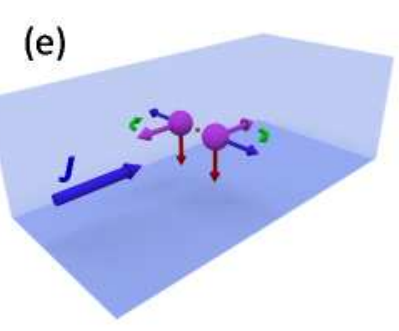

(f)

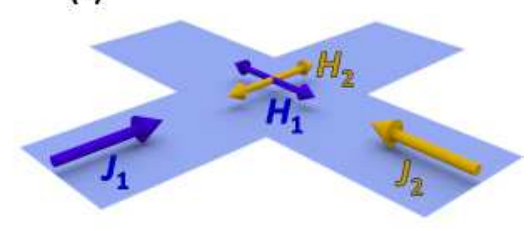

Fig. 1. Illustration of the current-induced effective magnetic field $\boldsymbol{H}$ and spin-orbit torque $\boldsymbol{T}$ acting on a magnetic moment $\boldsymbol{m}(\mathrm{a}, \mathrm{b})$ or a pair of antiferromagnetically coupled moments (c-e). (a,c) Fieldlike torque; (b,d) Damping-like torque; (e) Néel-order field-like torque for magnetic moments which are inversion partners about the point labelled by an orange sphere. For the field-like torques, the effective field is along the direction given by the local current-induced spin polarization $\sigma$, while for the damping-like torques it is along the direction given by $\boldsymbol{m} \times \boldsymbol{\sigma}$. The green curved arrows indicate the direction of rotation of the magnetic moments. (f) Scheme for switching the AF staggered magnetization between two orthogonal easy axes using the Néel-order fields $\boldsymbol{H}_{\mathbf{1}}$ and $\boldsymbol{H}_{\mathbf{2}}$ induced by currents $\boldsymbol{J}_{\mathbf{1}}$ and $\boldsymbol{J}_{\mathbf{2}}$ applied along orthogonal directions.

\section{Properties of tetragonal CuMnAs films}

The tetragonal phase of the collinear antiferromagnet CuMnAs has the magnetic structure shown in Fig. 2(a). The space group is that of the tetragonal $\mathrm{Cu}_{2} \mathrm{Sb}$ family, $\mathrm{P} 4 / \mathrm{nmm}$. Due to the strong magnetocrystalline anisotropy, the local magnetic moments align in the $a b$ plane up to the Néel temperature of $485 \mathrm{~K}^{[37]}$. The Mn spin sublattices form inversion partners, fulfilling the symmetry requirement for a current-induced NSOT. 
(a)

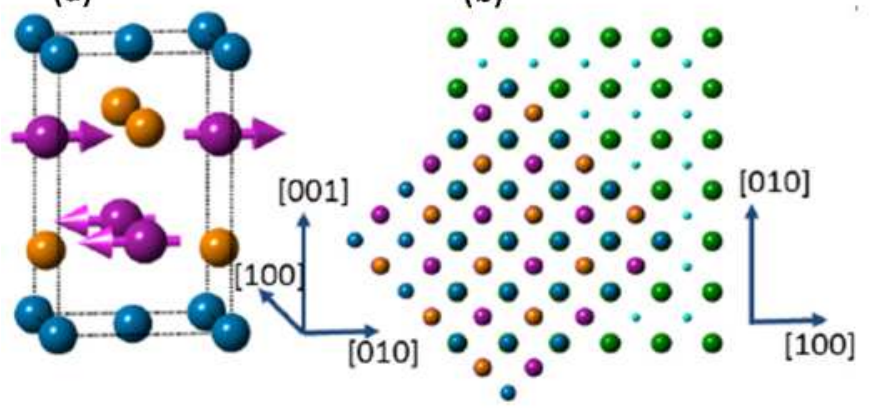

(d)

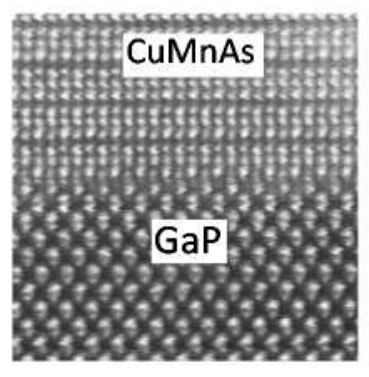

(b)

(e)

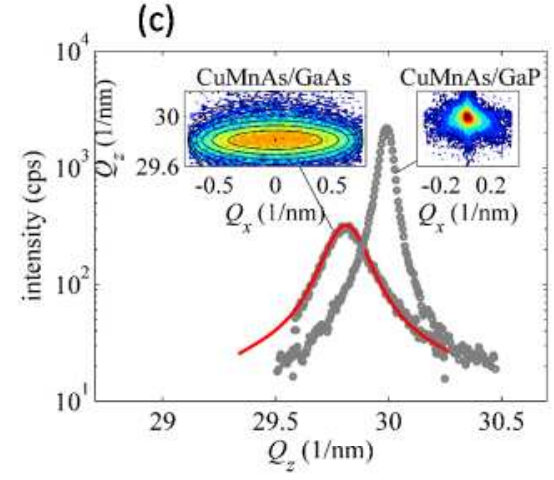

(f)
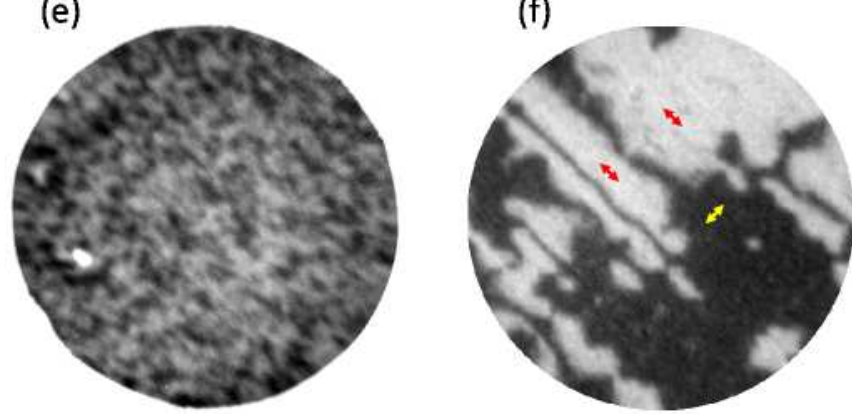

Fig. 2. (a) Unit cell structure and spin arrangement of tetragonal CuMnAs, with $\mathrm{Mn}$ (purple), $\mathrm{Cu}$ (blue) and As( orange) (from Ref. [40]). (b) Registry of the CuMnAs with a III-V semiconductor substrate (from Ref. [40]). (c) X-ray $2 \theta-\omega$ scans (main figure) and reciprocal space maps (inset) of the CuMnAs(003) diffraction peak for layers on $\mathrm{GaP}$ and GaAs (from Ref. [40]). (d) Z-contrast scanning transmission electron microscopy image for the (100) plane of CuMnAs on $\mathrm{GaP}(001)$ (from Ref. [3]). (e), (f) $10 \mu \mathrm{m}$ field-of-view x-ray photoemission electron microscopy images of antiferromagnetic domains in (e) 80nm CuMnAs on GaAs(001) (adapted from Ref. [4]) and (f) 45nm CuMnAs on GaP(001) (adapted from Ref. [7]).

The stable bulk phase of CuMnAs has an orthorhombic structure ${ }^{[38,39]}$, but the tetragonal phase can be stabilized by epitaxial growth on III-V semiconductor substrates ${ }^{[40]}$. The $a b$ plane is lattice-matched to the substrate through a $45^{\circ}$ rotation as illustrated in Fig. 2(b). The $a b$ lattice parameter of $3.82 \AA$ is mismatched to $\mathrm{GaP}(001)$ along the diagonal by only $\approx 0.5 \%$. X-ray diffraction (XRD) linescans and reciprocal space maps (Fig. 2(c)) indicate that the layer remains coherently strained on $\mathrm{GaP}(001)$ for thicknesses of several 10s of nm. In contrast, for growth on $\mathrm{GaAs}(001)$, strain relaxation is observed after only a few monolayers growth, and the resulting broadening of the reciprocal space map is described by a mosaic block model with lateral dimensions $\approx 50 \mathrm{~nm}$. Z-resolved scanning transmission electron microscopy images (Fig. 2(d)) indicate a high degree of structural and chemical order of the films.

The bulk and interface structure has a marked effect on the AF order. For CuMnAs films of thickness $\approx 10 \mathrm{~nm}$ or lower, the interface anisotropy results in a uniaxial easy magnetic axis parallel to the substrate [110] crystal axis ${ }^{[37,41]}$. Similar uniaxial magnetic anisotropies, originating from the reduced symmetry of the bonding at the III-V semiconductor surface, have been studied in detail for the Fe/GaAs(001) system ${ }^{[42]}$. 
With increasing thickness, the uniaxial interface anisotropy becomes progressively less important, and the tetragonal anisotropy of the bulk crystal becomes dominant. However, the magnetic domain microstructure, measured using x-ray photoemission electron microscopy (XPEEM), is strongly dependent on the substrate. For CuMnAs films grown on $\mathrm{GaAs}(001)$, an inhomogeneous AF domain structure is observed (Fig. 2(e)), with no evident preference for a particular in-plane axis, and with length scales down to the spatial resolution of the measurement $(\approx 50 \mathrm{~nm})$. This is likely to be related to a distribution of local strains in the film, consistent with the mosaic block structure revealed by XRD. For fully strained CuMnAs on $\mathrm{GaP}(001)$, a biaxial magnetic anisotropy is observed. The domains, with typical sizes of several micrometres, are found to have their spin axis aligned along one of the in-plane $\langle 110\rangle$ axes of the CuMnAs film, and are separated by $90^{\circ}$ domain walls (Fig. 2(f)).

Recent theoretical studies have shown that the orthorhombic phase of CuMnAs also possesses a lattice symmetry favouring efficient switching by NSOT ${ }^{[43]}$. Moreover, the lower symmetry of this structure is predicted to favour a Dirac semimetallic band structure at the Fermi level ${ }^{[43,44]}$, opening up the intriguing possibility of combining antiferromagnetic spintronics with topological Dirac physics. However, growth of orthorhombic CuMnAs thin films has not yet been reported, to our knowledge.

\section{Néel-order spin-orbit torque in CuMnAs}

The experimental geometry used in the first demonstration of current-induced switching in CuMnAs is shown in Fig. 3(a) ${ }^{[3]}$. Electrical current pulses are directed along the main axes of the 8-arm device, producing a current-induced Néel-order effective magnetic field perpendicular to the current. The diagonal arms allow electrical probing of the resistance anisotropy, which depends on the orientation of the antiferromagnetic spins. In the presence of either a FM or AF spin axis, the longitudinal and transverse components of the electrical resistance are given, respectively, by

$$
\begin{aligned}
& R_{/ /}=R_{0}+R_{1} \cos (2 \phi) \\
& R_{\perp}=R_{1} \sin (2 \phi)
\end{aligned}
$$

where $\phi$ is the angle between the spin axis and the current direction, and $R_{0}$ and $R_{1}$ are constants. Therefore, a current-induced rotation of the AF spin axis in the direction of the NSOT effective field will produce a change in $R_{\perp}$ measured in the geometry of Fig. 3(a), with an opposite sign of $R_{\perp}$ following current pulses along the [100] and [010] directions. The resulting highly reproducible behaviour of $R_{\perp}$ following orthogonal current pulses is shown in Fig. 3(b). Further measurements for probing currents applied along different arms of the device confirmed the expected symmetry of Eq. 3 and Eq. 4 for the resistance components following each current pulse. 

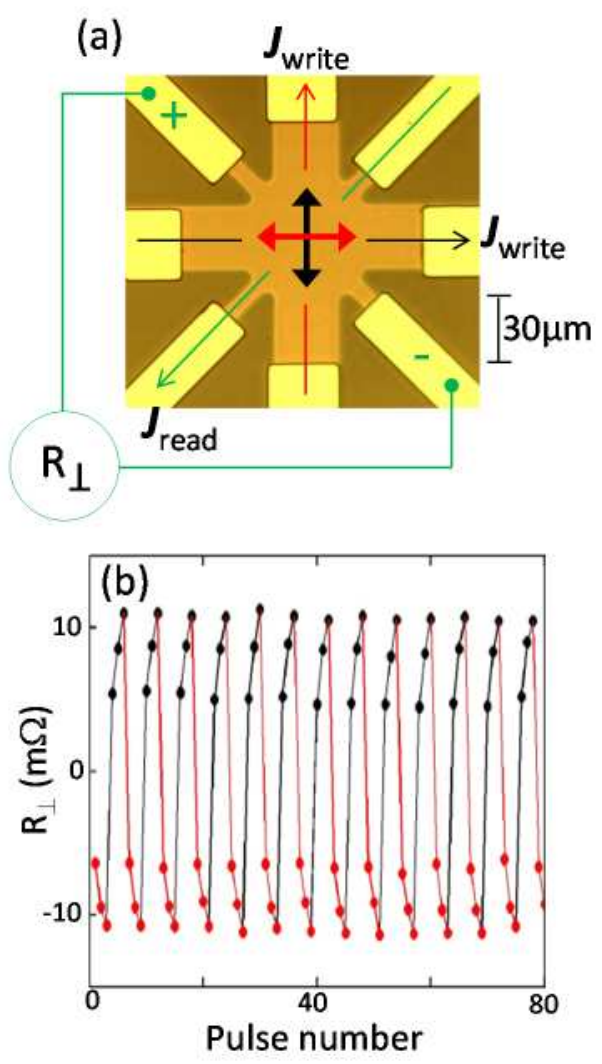

Fig. 3. (a) Optical microscopy of an 8-arm CuMnAs device, and schematic of the electrical pulsing and measurement geometry. (b) Change in the transverse resistance after applying three successive writing pulses of amplitude $J_{\text {write }}=4 \times 10^{6} \mathrm{Acm}^{-2}$ along the [100] axis (black arrow in (a) and black points in (b)) and along the [010] axis (red arrow in (a) and red points in (b)). Adapted from Ref. [3].

The current-induced switching of AF domains into the NSOT effective field direction was confirmed by XPEEM imaging ${ }^{[4]}$. For an $80 \mathrm{~nm}$ thick CuMnAs layer on GaAs(001), the AF moments were shown to be, on average, rotated to be perpendicular to the direction of the current pulse. A clear correlation was observed between the average spin orientation and the anisotropy of the electrical resistance, measured in-situ after each pulse. However, the observed switching was found to be inhomogeneous at the submicron scale. Small localized regions were found to consistently and reversibly switch with each current pulse, while other regions switch erratically or not at all. This is indicative of a stochastic process, highlighting the important role of thermal fluctuations and a distribution of domain wall pinning centres.

As can be seen in Fig. 3(b), successive current pulses applied along the same direction result in a steady increase of the readout signal. This multi-level behaviour was investigated in detail in Ref. ${ }^{[5]}$. It was found that the readout signal reaches a reproducible level for a given number of pulses or total pulse duration, suggesting potential pulse counting, integration or multi-level memory applications. This reproducible monotonic behaviour of the readout signal was observed for current pulses 
varying from $1 \mathrm{~ms}$ to $250 \mathrm{ps}$, and is ascribed to the multi-domain nature of the switching. The study was subsequently extended to the $\mathrm{THz}$ regime ${ }^{[6]}$, where a remarkable consistency was observed between the effects of microsecond and picosecond pulses (Fig. 4).
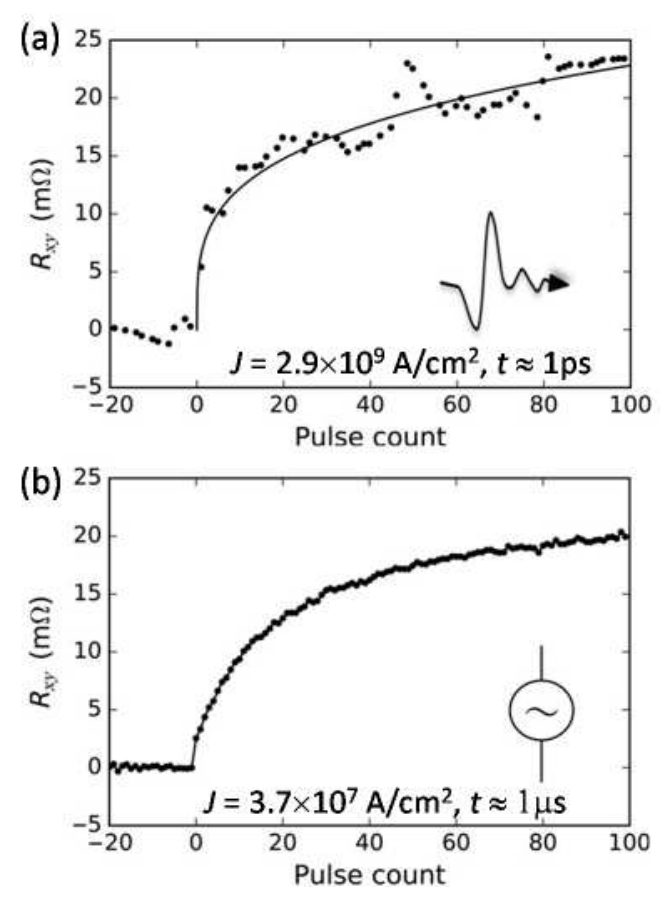

Fig. 4. (a) Output signal of a $2 \mu \mathrm{m} \mathrm{CuMnAs} \mathrm{bit} \mathrm{cell} \mathrm{as} \mathrm{a} \mathrm{function} \mathrm{of} \mathrm{the} \mathrm{number} \mathrm{of} \mathrm{applied} \mathrm{picosecond}$ pulses of amplitude $2.9 \times 10^{9} \mathrm{Acm}^{-2}$. (b) Same as (a), but for microsecond pulses of amplitude $3 \times 10^{7}$ $\mathrm{Acm}^{-2}$ applied to a $3.5 \mu \mathrm{m}$ CuMnAs bit cell. Adapted from Ref. [6].

The switching due to current induced by $\mathrm{THz}$ radiation relies on the fact that electrical readout signal depends on the axis of the applied current pulse but not the polarity. Changing the polarity of the pulse reverses the sign of the spin-orbit torque, but the readout described by eqn. (4) is not sensitive to $180^{\circ}$ rotated states. Therefore, a.c. and d.c. currents can be equally effective in rotating the antiferromagnetic spin axis. On the other hand, for a $45 \mathrm{~nm}$ thick CuMnAs layer on $\mathrm{GaP}(001)$, in certain geometries, a clear dependence on the polarity of the current pulse was observed ${ }^{[7]}$. The layer exhibits micron-sized biaxial magnetic domains, similar to the ones shown in Fig. 2(f), and the current is applied at $45^{\circ}$ to the spin axis. The Néel-order effective magnetic field produces a difference in the energy density between neighbouring domains, resulting in a force on the domain wall in the direction of the energetically less favoured domain. It was shown using both electrical transport measurements and XPEEM imaging that a reversal of the current polarity can induce a $90^{\circ}$ switch of the spin axis within an AF domain, driven by the current-induced motion of an AF domain wall (Fig. 5) ${ }^{\text {[7] }}$. Switching by domain wall propagation typically occurs at lower threshold current densities than required for coherent domain switching; for example, switching by domain wall nucleation and propagation was recently demonstrated for $500 \mathrm{~nm}$ Co disks with 100 ps time resolution ${ }^{[45]}$. 

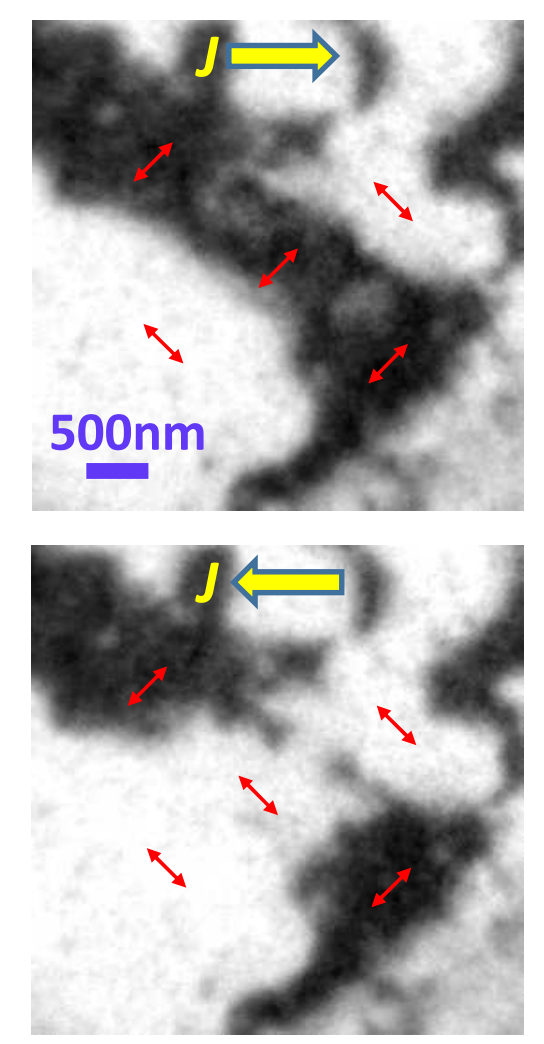

Fig. 5. XPEEM images showing current-induced writing of an antiferromagnetic domain in CuMnAs. The orientation of the staggered magnetization in the domain (red double-headed arrows) is switched by $90^{\circ}$ when the polarity of the current pulse is reversed. Adapted from Ref. [7].

Current-induced switching of $180^{\circ}$ domains was recently demonstrated using a novel phase-sensitive detection technique ${ }^{[8]}$. The technique relies on the fact that the currentinduced local non-equilibrium spin polarization has opposite sign on the two magnetic sublattices. A current applied parallel to the uniaxial easy axis results in a transient rotation of the local moments towards the direction of the Néel-order effective magnetic field (Fig. 6(a)). Switching the magnetic sublattices reverses the handedness of the rotation (Fig. 6(b)). The resulting transient rotations can be detected using 2ndharmonic transport measurements. The experiment, performed on a CuMnAs(10nm) / $\mathrm{Pt}(3 \mathrm{~nm})$ bilayer, indicated that a $180^{\circ}$ spin reversal could be induced reproducibly by changing the polarity of the writing current pulse. It also indicated that the multi-level switching observed for $90^{\circ}$ rotation is not evident for $180^{\circ}$ switching, and also that the resulting domain configuration may be more stable. 

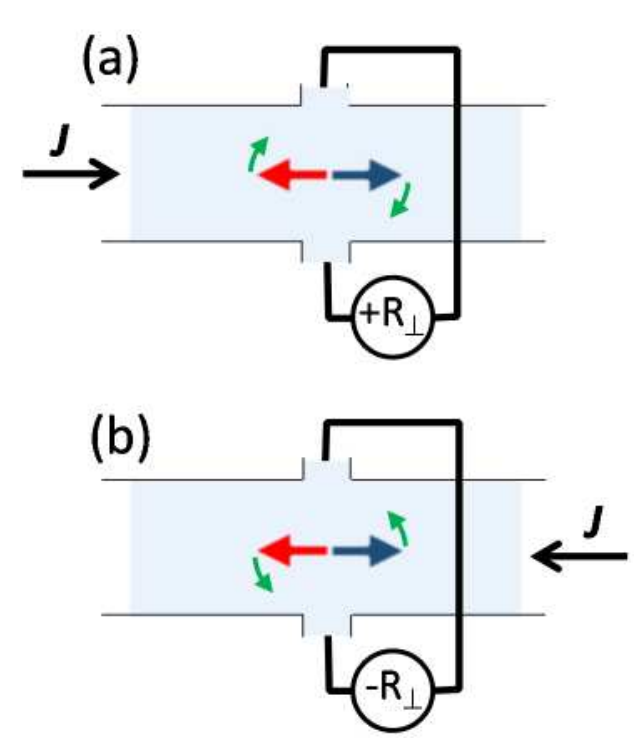

Fig. 6. Experimental geometry used to detect the polarity of $180^{\circ}$ antiferromagnetic domains. The AF easy axis is oriented along the Hall bar. The staggered effective field due to an applied current results in a transient rotation of the spin axis, in a direction that depends on the relative orientations of the sublattice magnetization and the current. The two rotation directions result in opposite sign of the transverse resistance $R_{\perp}$, detected using $2^{\text {nd }}$ harmonic lock-in methods [8].

\section{Current-induced switching in $\mathrm{Mn}_{2} \mathrm{Au}$ and $\mathrm{Pt} / \mathrm{NiO}$}

The existence of current-induced Néel-order fields was first predicted for $\mathrm{Mn}_{2} \mathrm{Au}$, a collinear AF in which, as with CuMnAs, the Mn magnetic sublattices are inversion partners in an otherwise centrosymmetric crystal ${ }^{[31]}$. Its high magnetic ordering temperature, well above $1000 \mathrm{~K}$, makes this a promising material for spintronics applications ${ }^{[46]}$. Using a similar geometry to the one shown in Fig. 3(a) (ref. [3]), consistent and reversible current-induced changes of the longitudinal and transverse resistances were recently demonstrated in epitaxial $\mathrm{Mn}_{2} \mathrm{Au}(001)$ films ${ }^{[9]}$. Large readout signals, of up to $6 \%$ of the longitudinal resistivity, were observed. Elsewhere, investigations of similarly sputtered $\mathrm{Mn}_{2} \mathrm{Au}$ films pointed to an important role of thermal activation in the current-induced reorientation process of the AF domains ${ }^{[10]}$.

Zhou et al. investigated the NSOT-induced switching in $\mathrm{Mn}_{2} \mathrm{Au}$ with the current applied along different crystal axes ${ }^{[11]}$. By growth on either $\mathrm{MgO}(111), \mathrm{MgO}(110)$ or SrTiO3(100) substrates, $\mathrm{Mn}_{2} \mathrm{Au}$ films with (103), (204) and (101) were obtained. The different switching behaviours observed for each film, combined with x-ray measurements of the crystal anisotropy, indicate that the AF moments are switched between the easy [010] and [100] axes, the latter of which lies out of the plane. The observed orientation-dependent NSOT points to the important role of the magnetocrystalline anisotropy in the current-induced switching process. 


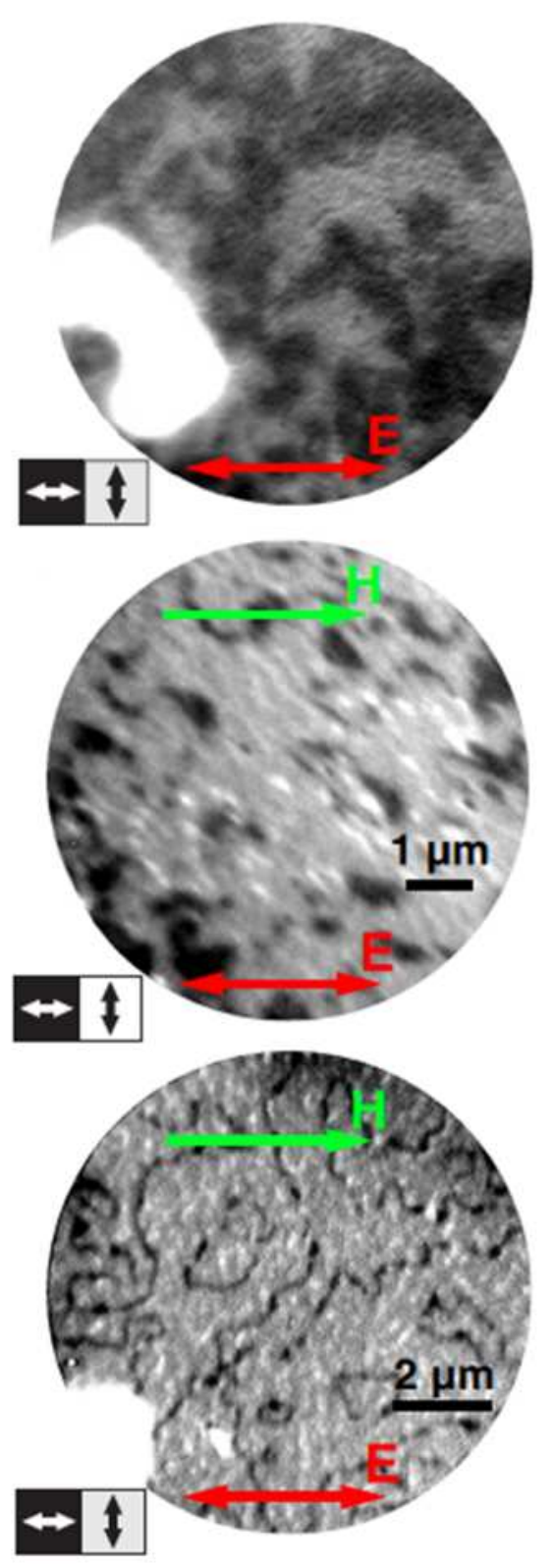

Fig. 7. XPEEM images of the antiferromagnetic domain structure of a $\mathrm{Mn}_{2} \mathrm{Au}$ film in its initial state (a), and after applying magnetic field pulses of 30T (b) and 50T (c) along one of the biaxial in-plane easy axes. Adapted from Ref. [47].

AF domain images obtained using XPEEM also indicate a substantial magnetocrystalline anisotropy in $\mathrm{Mn}_{2} \mathrm{Au}$ films ${ }^{[47]}$. Measurements of as-prepared $\mathrm{Mn}_{2} \mathrm{Au}(001)$ films revealed a biaxial magnetic anisotropy, with approximately equal populations of domains with spin oriented along the in-plane [110] and [11̄0] axes. After subjecting the films to external magnetic fields of between 30 and 50T along the [110] direction, domains were observed to be predominantly oriented along [1 $\overline{1} 0]$, indicative of a spin flop transition (Fig. 7). The high magnetic field required to induce the spin flop transition, compared with the $\sim 1 \mathrm{mT}$ Néel-order effective field required for current-induced switching ${ }^{[9]}$, highlights the efficiency of the Néel-order spin-orbit torque mechanism. 
$\mathrm{THz}$ spectroscopy measurements of $\mathrm{Mn}_{2} \mathrm{Au}$ films revealed a strong mode near $1 \mathrm{THz}$, ascribed to a resonant in-plane oscillation of the AF coupled magnetic moments ${ }^{[48]}$. The absorption strength of the AF magnetic resonance is some 3 orders of magnitude larger than in insulating AF materials such as $\mathrm{NiO}$, where the magnetic field component

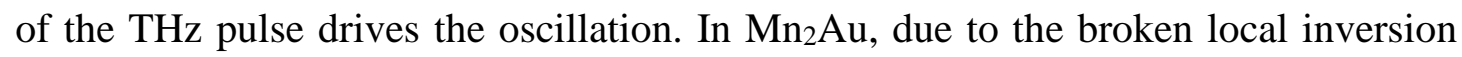
symmetry, the electric component of the pulse induces a Néel-order effective magnetic field, which can account for the observed strong AF magnetic resonance.

Current-induced switching behaviour was also demonstrated in bilayer films of $\operatorname{Pt}(5 \mathrm{~nm})$ / $\mathrm{NiO}(5 \mathrm{~nm}){ }^{[12]}$. While the measurement geometry as well as the critical current densities were similar to the earlier studies of $\mathrm{CuMnAs}^{[3]}$ and $\mathrm{Mn}_{2} \mathrm{Au}^{[9,10]}$, there are crucial differences in the underlying mechanism. Firstly, the longitudinal and transverse resistance changes were ascribed to the spin Hall magnetoresistance effect in the $\mathrm{Pt}$ layer ${ }^{[49]}$. More importantly, the $\mathrm{NiO}$ film does not possess the broken sublattice inversion symmetry required for a Néel-order field-like torque. Instead, the switching is ascribed to the damping-like torque due non-equilibrium spin polarization generated in the $\mathrm{Pt}$ layer. The opposite sign of the resistance change in $\mathrm{Pt} / \mathrm{NiO}$ compared to $\mathrm{Mn}_{2} \mathrm{Au}$ is consistent with this, and indicates that the AF-coupled local moments tend to switch into the axis of the current pulse. Some aspects of the observed switching behaviour are unclear, particularly the role of the AF domain microstructure in the quasiepitaxial $\mathrm{NiO}(001)$ film. However, the observed switching in a heavy-metal / AF bilayer points to a host of new possibilities for current-induced manipulation and high speed dynamics in AF thin films, without the requirement for a particular crystal symmetry.

Table 1 summarizes the range of writing current densities and pulse widths explored in CuMnAs, $\mathrm{Mn}_{2} \mathrm{Au}$ and $\mathrm{Pt} / \mathrm{NiO}$ memory devices for which stable and reproducible switching of the AF order is reported.

\begin{tabular}{|l|l|l|l|}
\hline Material & $t(\mathrm{~s})$ & $J_{\text {write }}\left(\mathrm{Acm}^{-2}\right)$ & ref. \\
\hline $\mathrm{CuMnAs}$ & $0.005-0.05$ & $(4.0-4.5) \times 10^{6}$ & 3 \\
\hline & 0.05 & $(5.7-6.1) \times 10^{6}$ & 4 \\
\hline & $10^{-3}-2.5 \times 10^{-10}$ & $(2-16) \times 10^{7}$ & 5 \\
\hline & $\approx 10^{-12}$ & $2.9 \times 10^{9}$ & 6 \\
\hline & 0.05 & $5 \times 10^{5}$ & 7 \\
\hline $\mathrm{Mn}_{2} \mathrm{Au}$ & $10^{-3}$ & $(1.4-1.8) \times 10^{7}$ & 9 \\
\hline & $2 \times 10^{-4}$ & $2 \times 10^{7}$ & 10 \\
\hline & $10^{-3}$ & $2 \times 10^{7}$ & 11 \\
\hline $\mathrm{Pt} / \mathrm{NiO}$ & $10^{-3}$ & $4 \times 10^{7}$ & 12 \\
\hline
\end{tabular}

Table 1. Current pulse width $(t)$ and amplitude $\left(J_{\text {write }}\right)$ used for switching of AF domains in CuMnAs, $\mathrm{Mn}_{2} \mathrm{Au}$ and $\mathrm{Pt} / \mathrm{NiO}$. 


\section{Conclusion and perspectives}

The recent rapid growth and development of antiferromagnetic spintronics is in large part associated with the discovery and demonstration of electrical switching in CuMnAs using NSOT. This offers an efficient and reversible method to control AF coupled spins, providing new possibilities for manipulating AF domains and domain walls and understanding their dynamical properties. Since then the demonstration of reproducible multi-level switching and response to terahertz pulses opens a pathway to applications in fields such as ultrafast processing and neuromorphic logic.

The demonstration of electrical current control in other AF material systems has broadened the material base for AF spintronics. Particularly the demonstration of switching in bilayer films, which are not limited by the same symmetry constraints, opens a new plethora of potential AF material systems.

Obstacles to application certainly exist and include the small size of the read-out signal using ohmic anisotropic magnetoresistance. The further development of AF tunneling anisotropic magnetoresistance structures would be of huge benefit. Another predicted property of some AF materials, including CuMnAs, could help to remedy this in a novel way. The predictions of the existence of a topological metal-insulator transition linked to the magnetic order ${ }^{[42]}$ could provide a means of producing a large readout signal. As of yet the existence of this transition has not been demonstrated.

A further obstacle to application is the lack of understanding of what dictates the domain formation in these materials. The ability to tailor the domain size and anisotropies would allow one to design stable, single domain memory devices as well as to electrically manipulate individual AF domain walls. This would enable future spintronic devices utilizing AF domain wall motion induced by ultrafast current pulses.

\section{References}

1. Hellman F, Hoffman A, Tserkovnyak Y et al. 2017 Rev. Mod. Phys. 89025006

2. Park B G, Wunderlich J, Marti X, Holy V, Kurosaki Y, Yamada M, Yamamoto H, Nishide A, Hayakawa J, Takahashi H, Shick A B and Jungwirth T 2011 Nat. Mater. 10347

3. Wadley P, Howells B, Zelezny J et al. 2016 Science 351587

4. Grzybowski M J, Wadley P, Edmonds K W, Beardsley R, Hills V, Campion R P, Gallagher B L, Chauhan J S, Novak V, Jungwirth T, Maccherozzi F and Dhesi S S 2017 Phys. Rev. Lett. 118 057701

5. Olejník K, Schuler V, Marti X, Novák V, Kašpar Z, Wadley P, Campion R P, Edmonds K W, Gallagher B L, Garces J, Baumgartner M, Gambardella P and Jungwirth T 2017 Nat. Commun. 815434

6. Olejnik K, Seifert T, Kašpar Z, Novák V, Wadley P, Campion R P, Baumgartner M, Gambardella P, Němec P, Wunderlich J, Sinova J, Kužel P, Müller M, Kampfrath T and Jungwirth T 2018 Science Adv. 4 eaar3566

7. Wadley P, Reimers S, Grzybowski M J, Andrews C, Wang M, Chauhan J S, Gallagher B L, 
Campion R P, Edmonds K W, Dhesi S S, Maccherozzi F, Novak V, Wunderlich J and Jungwirth T 2018 Nat. Nanotechnol. 13362

8. Godinho J, Reichlova H, Kriegner D, Novak V, Olejnik K, Kaspar Z, Soban Z, Wadley P, Campion R P, Otxoa R M, Roy P E, Zelezny J, Jungwirth T and Wunderlich J 2018 arXiv: 1806.02795 [cond-mat]

9. Bodnar S Yu, Šmejkal L, Turek I, Jungwirth T, Gomonay O, Sinova J, Sapozhnik A A, Elmers H-J, Kläui M and Jourdan M 2018 Nat. Commun. 9348

10. Meinert M, Graulich D, and Matalla-Wagner T 2018 Phys. Rev. Applied 9, 064040

11. Zhou X F, Zhang J, Li F, Chen X Z, Shi G Y, Tan Y Z, Gu Y D, Saleem M S, Wu H Q, Pan F and Song C 2018 Phys. Rev. Applied 9054028

12. Chen X Z, Zarzuela R, Zhang J, Song C, Zhou X F, Shi G Y, Li F, Zhou H A, Jiang W J, Pan F and Tserkovnyak Y 2018 Phys. Rev. Lett. 120207204

13. Kittel C 1951 Phys. Rev. 85565

14. Kimel A V, Kirilyuk A, Tsvetkov A, Pisarev R V and Rasing Th 2004 Nature 429850

15. Editorial 2018 Nat. Phys. 14199 and references therein.

16. Jungwirth T, Marti X, Wadley P and Wunderlich J 2016 Nat. Nanotechnol. 11231

17. Baltz V, Manchon A, Tsoi M, Moriyama T, Ono T and Tserkovnyak Y 2018 Rev. Mod. Phys. 90 015005

18. Chernyshov A, Overby M, Liu X, Furdyna J K, Lyanda-Geller Y and Rokhinson L P 2009 Nat. Phys. 5656

19. Ciccarelli C, Anderson L, Tshitoyan V, Ferguson A J, Gerhard F, Gould C, Molenkamp L W, Gayles J, Železný J, Šmejkal L, Yuan Z, Sinova J, Freimuth F and Jungwirth T 2016 Nat. Phys. 12855

20. Miron I M, Garello K, Gaudin G, Zermatten P-J, Costache M V, Auffret S, Bandiera S, Rodmacq B, Schuhl A and Gambardella P 2011 Nature 476189

21. Garello K, Miron I M, Avci C O, Freimuth F, Mokrousov Y, Blugel S, Auffret S, Boulle O, Gaudin G and Gambardella P 2013 Nat. Nanotechnol. 8587

22. Liu L, Moriyama T, Ralph D C, and Buhrman R A 2011 Phys. Rev. Lett. 106036601

23. Liu L, Pai C-F, Li Y, Tseng H W, Ralph D C and Buhrman R A 2012 Science 336555

24. Fukami S, Anekawa T, Zhang C and Ohno H 2016 Nat. Nanotechnol. 11621

25. Yu G, Upadhyaya P, Fan Y, Alzate J G, Jiang W, Wong K L, Takei S, Bender S A, Chang L-T and Jiang Y 2014 Nat. Nanotechnol. 9548

26. Oh Y-W, Baek S C, Kim Y, Lee H Y, Lee K D, Yang C D, Park E S, Lee K S, Kim K W, Go G, Jeong J-R, Min B C, Lee H-W, Lee K-J and Park B-G 2016 Nat. Nanotechnol. 11878

27. Cai K, Yang M, Ju H, Wang S, Ji Y, Li B, Edmonds K W, Sheng Y, Zhang B, Zhang N, Liu S, Zheng H and Wang K Y 2017 Nat. Mater. 16712

28. Baek S C, Amin V P, O Y-W, Go G, Lee S-J, Lee G-H, Kim K-J, Stiles M D, Park B-G and Lee K-J 2018 Nat. Mater. 17509

29. Gomonay H V and Loktev V M 2010 Phys. Rev. B 81144427

30. Zhang X, Liu Q, Luo J-W, Freeman A J and Zunger A 2014 Nat. Phys. 10387

31. Zelezny J, Gao H, Vyborny K, Zemen J, Masek J, Manchon A, Wunderlich J, Sinova J and Jungwirth T 2014 Phys. Rev. Lett. 113157201

32. Zelezny J, Gao H, Manchon A, Freimuth F, Mokrousov Y, Zemen J, Masek J, Sinova J and Jungwirth T 2017 Phys. Rev. B 95014403 
33. Roy P E, Otxoa R M and Wunderlich J 2016 Phys. Rev. B 94014439

34. Shiino T, Oh S-H, Haney P M, Lee S-W, Go G, Park B-G and Lee K-J 2016 Phys. Rev. Lett. 117087203

35. Gomonay O, Jungwirth T and Sinova J 2016 Phys. Rev. Lett. 117017202

36. Schryer N L and Walker L R 1974 J. Appl. Phys. 455406

37. Wadley P, Hills V, Shahedkhah M R, Edmonds K W, Campion R P, Novak V, Ouladdiaf B, Khalyavin D, Langridge S, Saidl V, Nemec P, Rushforth A W, Gallagher B L, Dhesi S S, Maccherozzi F, Zelezny J and Jungwirth T 2015 Sci. Rep. 517079

38. Mundelein J and Schuster H U 1992 Zeit. Naturforschung B 47925

39. Máca F, Mašek J, Stelmakhovych O, Martí X, Reichlová H, Uhlírová K, Beran P, Wadley P, Novák V and Jungwirth T 2012 J. Magn. Magn. Mater. 3241606

40. Wadley P, Novak V, Campion R P et al. 2013 Nat. Commun. 42322

41. Saidl V, Nemec P, Wadley P, Hills V, Campion R P, Novak V, Edmonds K W, Maccherozzi F, Dhesi S S, Gallagher B L, Trojanek F, Kunes J, Zelezny J, Maly P and Jungwirth T 2017 Nat. Photon. 1191

42. Wastlbauer G and Bland J A C 2005 Adv. Phys. 54137

43. Šmejkal L, Železný J, Sinova J and Jungwirth T 2017 Phys. Rev. Lett. 118106402

44. Tang P, Zhou Q, Xu G and Zhang S C 2016 Nat. Phys. 121100

45. Baumgartner M, Garello K, Mendil J, Avci CO, Grimaldi E, Murer C, Feng J, Gabureac M, Stamm C, Acremann Y, Finizio S, Wintz S, Raabe J and Gambardella P 2017 Nat. Nanotechnol. $\underline{12980}$

46. Barthem V M T S, Colin C V, Mayaffre H, Julien M-H and Givord D 2013 Nat. Commun. 4 2892

47. Sapozhnik A A, Filianina M, Bodnar S Yu, Lamirand A, Mawass M-A, Skourski Y, Elmers HJ, Zabel H, Kläui M and Jourdan M 2018 Phys. Rev. B 97134429

48. Bhattacharjee N, Sapozhnik A A, Bodnar S Yu, Grigorev V Yu, Agustsson S Y, Cao J, Dominko D, Obergfell M, Gomonay O, Sinova J, Kläui M, Elmers H-J, Jourdan M. and Demsar J 2018 Phys. Rev. Lett. 120237201

49. Nakayama H, Althammer M, Chen Y T, Uchida K, Kajiwara Y, Kikuchi D, Ohtani T, Geprägs S, Opel M, Takahashi S, Gross R, Bauer G E W, Goennenwein S T B and Saitoh E 2013 Phys. Rev. Lett. 110206601 